\title{
Evaluating a Novel Multifactorial Falls Prevention Activity Programme for Community-Dwelling Older People After Stroke: A Mixed-Method Feasibility Study
}

This article was published in the following Dove Press journal: Clinical Interventions in Aging

Jun Sheng Gary Koh (D) Anne-Marie Hill (D)

Keith D Hill (D) ${ }^{1,2}$

Christopher EthertonBeer (D) ${ }^{3}$ Jacqueline Francis-Coad (D) Elizabeth Bell (D)

Liz Bainbridge $\mathbb{D}^{\prime}$ Lex $D$ de Jong $\mathbb{D}^{\prime}$

'School of Physiotherapy and Exercise Science, Faculty of Health Sciences, Curtin University, Bentley, Perth, WA 6102, Australia; ${ }^{2}$ Rehabilitation, Ageing and Independent Living Research Centre, Monash University, Melbourne, Victoria, Australia; ${ }^{3}$ WA Centre for Health and Ageing, Royal Perth Hospital Unit, School of Medicine and Pharmacology and Centre for Medical Research, University of Western Australia, Perth, Western Australia, Australia
Correspondence: Lex D de Jong School of Physiotherapy and Exercise Science, Faculty of Health Sciences, Curtin University, Bentley, Perth, WA 6102, Australia

Email lex.dejong@curtin.edu.au
Purpose: The overall purpose of this study was to explore participants' and physiotherapists' experiences regarding the acceptability, implementation, and practicality of a novel group-based multifactorial falls prevention activity programme for community-dwelling older people after stroke. Specifically, the purpose was to explore if and how participating could impact on the participants' health-related quality of life (HRQoL) in terms of their daily lived experience regarding physical, mental, emotional and social well-being. A secondary purpose was to explore whether participating in the programme could positively influence participants' balance, strength, falls efficacy, mobility and motor impairment of the trunk.

Materials and Methods: This was an exploratory mixed-method Phase I feasibility study. A convenience sample of five older community-dwelling people after stroke participated in a novel eight-week multifactorial activity programme which included falls education, a mix of individually tailored and group-based strength and balance exercises, exploring limits of stability and safe landing techniques and a social element. Qualitative data from post-intervention interview transcripts with the participants and the physiotherapists who delivered the programme were thematically analysed using both deductive and inductive approaches to explore the participants' and therapists' experiences with the programme. Quantitative outcomes included balance, strength, falls efficacy, mobility and motor impairment of the trunk.

Results: The programme was deemed feasible in terms of acceptability, implementation and practicality by the participants as well as the physiotherapists delivering the programme. The overarching theme regarding HRQoL identified that participating in the programme was perceived to empower the participants living with stroke and positively influenced their daily physical, mental, emotional and social well-being. Participant outcomes showed a change in the direction of improvements in balance, strength, mobility, motor impairment of the trunk and reduced concerns about falling. Subjectively, participants only reported perceived improvements in balance and strength.

Conclusion: Running a novel multifactorial falls prevention activity programme for older community-dwelling people after stroke was feasible. Participating in the programme helped participants to perceive improved balance, strength and empower them to make meaningful changes, improving their daily lived experiences. A future fully powered study could build on these results to investigate physical improvements, prevention of falls and improvements to domains of HRQoOL.

Keywords: accidental falls, aged, exercise, evaluation studies as topic, stroke, qualitative research, quality of life 


\section{Introduction}

Annually, 15 million people worldwide suffer a stroke ${ }^{1}$ and approximately 80 million people were living with stroke in 2016 alone. $^{2}$ Between $50 \%$ and $70 \%$ of stroke survivors experience residual deficits such as muscle weakness, sensory loss, and abnormalities of vision and spatial awareness. ${ }^{3}$ These falls-risk increasing impairments in body functions pose a significant challenge to maintaining balance and can impact on recovering meaningful functional activities such as gait and activities of daily life (ADL). Physical impairments can also negatively influence mental and psychosocial well-being, resulting in post-stroke depression, ${ }^{4}$ and an overall reduced healthrelated quality of life (HRQoL). ${ }^{5}$

People after stroke have a high risk of falling, with incidence rates reported to be as high as $45 \%$ to $73 \%$ in the chronic phase post-stroke. ${ }^{6,7}$ Due to ageing of the population and improvements in post-stroke life expectancy, the societal impact of falls after stroke is growing ${ }^{3}$ for both people after stroke and their carers. ${ }^{8}$ Falls may lead to physical harm such as hip fractures, ${ }^{9}$ an increased fear of falling ${ }^{10,11}$ and a subsequent decline in physical function and autonomy. ${ }^{12}$ This cascade of problems can hamper reintegration into society $^{13}$ and reduce social participation ${ }^{14}$ and quality of life (QoL). ${ }^{15}$

Exercise is a well-established evidence-based approach to falls prevention in community-dwelling older people ${ }^{16}$ and to improving balance capacity in people after stroke. ${ }^{17}$ Multifactorial approaches can also reduce the rate of falls ${ }^{18}$ in the general older population, but these are underexplored in patients after stroke. ${ }^{19}$ Examples of effective exercise interventions targeting strength and dynamic balance used within falls prevention services include the group-based Falls Management Exercise $(\mathrm{FaME})^{20,21}$ programme and the Otago $^{22}$ home exercise programme. A recent systematic review and meta-analysis has further provided evidence that gamified exercises ('Exergames') can improve balance and falls risk factors after stroke. ${ }^{23}$ Although it has been established that regular participation in physical activities and exercise is integral to reduce the risk of falls, ${ }^{24,25}$ other key elements such as falls prevention education, ${ }^{26}$ and home falls hazard evaluations and modifications ${ }^{27}$ may be no less important. Incorporating safe landing/falling techniques in falls prevention programmes as a means to teach individuals how to fall in such a manner to reduce injury may also help to reduce fear of falling and how seriously and frequently older people or people after stroke injure themselves from a fall. ${ }^{28}$
Research into the use of such safe landing/falls technique strategies by older adults is scarce. ${ }^{29,30}$ People after stroke also value the inclusion of a social element to programmes they participate in. ${ }^{31,32}$ Research into the use of these different strategies is warranted to verify the effectiveness and suitability for at-risk populations. ${ }^{28}$ Apart from the effects on physical and functional impairments resulting from participating in stroke-specific multifactorial falls prevention programmes, the effects on mental, emotional and social domains of well-being in stroke survivors remain unclear. ${ }^{33}$ The scant literature available has suggested that a community-based programme for stroke survivors including exercise, education and peer support could help participants to reconstruct their lives, for example in terms of increased physical and social confidence, thereby improving participation in their social world. ${ }^{34}$ This has led to the recommendation to include interventions addressing domains beyond physical impairments during stroke rehabilitation to optimise outcomes. ${ }^{14,34}$

Because people with residual impairments following a stroke are considered at increased risk of a fall, ${ }^{35}$ more and broader falls prevention research in this population is recommended. ${ }^{19,36}$ To this end, we developed a novel falls prevention activity programme that aimed to increase the participants' falls prevention knowledge, improve their balance and strength, evaluate their home falls hazards, and gain skills in how to land safely in case of a fall, move on the floor and get up again. The idea was to deliver the programme in a way that provided participants the opportunity to train and socially interact with support from a small group of peers. As a first step in identifying if this programme could work ${ }^{37,38}$ and to gather information to inform the design of a definitive study of its effectiveness, we set out to investigate its feasibility in a small group of community-dwelling older people after stroke. Adhering to guidelines for the design of feasibility studies, ${ }^{39}$ the primary aim of this study was to explore the participants' and physiotherapists' experiences regarding the acceptability, implementation, and practicality of the programme. The secondary aim was to test limitedefficacy by exploring how participating impacted on the participants' health-related quality of life (HRQoL) in terms of their daily lived experience regarding physical, mental, emotional and social well-being. A secondary purpose was to explore whether participating in the programme could positively influence participants' balance, strength, falls efficacy, mobility and motor impairment of the trunk. 


\section{Materials and Methods \\ Design}

This was an exploratory mixed-method Phase I feasibility study with a pre-post design.

\section{Ethical and Organizational Review}

The study was approved by Curtin University's Human Research Ethics Office (approval number HRE2018-0104). The trial was registered on March 19, 2018 (NCT03484351; https://clinicaltrials.gov/ct2/show/NCT03484351). Participant recruitment commenced on April 9, 2018. The last participant's follow-up assessment was on October 2, 2018.

\section{Participants}

A convenience sample of community-dwelling adults over the age of 50 and more than 6 months post-stroke from one urban area in Western Australia were invited to participate in the study. They were identified by physiotherapists (PTs) from the researchers' networks. Participants were eligible if they were living in the community, had good cognition (Mini Mental State Examination $\geq 25$ points) and were able to walk at least three times weekly outside their home without hands-on supervision (with or without the use of a walking aid; Functional Ambulation Classification ${ }^{40,41}$ category $\geq 4$ ). Participants with medical issues preventing them from participating in moderate to vigorous exercises, receptive aphasia impacting on the ability to follow instructions and who were unable to provide informed consent were excluded from participation. Written informed consent was obtained from all participants.

\section{Intervention}

The intervention (dubbed the Fall Monty Activity Programme) is a novel therapist-led group-based exercise and multifactorial falls prevention activity programme. For the purpose of this feasibility study, the programme was designed for older people after stroke to help prevent falls and aid in functional recovery by addressing limitations in strength and balance whilst also increasing social interaction and offering a platform to share thoughts about aspects contributing to their HRQoL. Twelve programme sessions were offered over an eight-week period. Each session comprised a recurring mix of established evidence-based activities and exercises as well as some new approaches. ${ }^{42}$ Details of the different activities and exercises are presented in Appendix 1. Each session was approximately 90-100 mins in duration, thus with full adherence the intervention comprised up to 18-20 hrs of exercise and education/ knowledge sharing. The sessions were organised in a room of approximately 6 by $12 \mathrm{~m}$ containing basic equipment such as foldable tables, chairs and a projector screen. Prior to each session exercise equipment was brought into the room such as crash mats, balance boards, exercise balls, Exergames hardware (laptop and motion tracking sensor), resistance bands and small exercise steps. The venue, which was located in a health and wellness centre of a university, was chosen for ease of public transport, parking and physical access. All programme sessions were delivered by two female qualified PTs who had three and over 20 years of experience with the treatment of people after stroke, respectively. They delivered the programme to a group of five participants. In order to also be able to supervise and guide some of the more challenging exercises on a one-to-one basis, this was considered the maximum group size. Where necessary, exercises and activities were tailored to, and performed at the discretion of, individual participants. A folder was issued to each participant to collect their educational information- and home exercise leaflets which were handed out in a staggered way after each session. Participants were asked to perform the selected home exercises for at least 10-20 mins on three weekdays for the duration of the programme. Participants self-reported details of the frequency and type of fall incidents during the programme period using a diary purposely designed for this study.

\section{Primary Outcomes}

A researcher who was experienced in qualitative research (LDdJ) and a physiotherapy student (JSGK) attended each programme session to take unstructured field notes using a reflective journal. They noted descriptions of participants' interactions, made general observations, and potential biases were noted to enhance trustworthiness, reflexivity and increase confirmability of the findings. ${ }^{43}$ They also conducted and noted informal conversations with participants during the classes to understand their experiences. Together they interviewed all participants individually about their experiences with the programme within 1 week after the final programme session. A semi-structured interview topic guide consisting of open-ended questions was created. For the participants, the questions were structured around four of eight recommended areas of focus for feasibility studies, ${ }^{39}$ ie acceptability, implementation, practicality and the intended effects ("limited efficacy") of the programme. Additionally, questions were structured around the domains of HRQoL 
(physical, mental, emotional and social well-being) as it was felt that there was limited knowledge about how a multifactorial falls prevention program could impact the daily lived experiences of stroke survivors. A broad framework was used to conceptualise how participants would react to participating in the novel programme. The two PTs who delivered the programme were also interviewed individually (by one researcher, LDdJ) within 1 week of the final programme session. Their interviews mainly focussed on the feasibility topics. The interview topics are presented in Table 1. All interviews were recorded, fully transcribed verbatim and anonymised by an external vendor. ${ }^{43}$ Transcripts were not returned to participants nor physiotherapists for comments or corrections.

\section{Secondary Outcomes}

All secondary (quantitative) outcomes were administered 1 week prior to the start of, and within 1 week after, the final programme session. Outcomes included validated and reliable falls research questionnaires Short Falls-Efficacy Scale-International (FES-I), ${ }^{44}$ European Quality of Life 5 Dimensions Health Questionnaire (EQ-5D-5L) ${ }^{45}$ and Stroke Impact Scale 3.0 (SIS). ${ }^{46,47}$ Three psychometrically strong balance and mobility tests administered were the Berg Balance Test (BBS), ${ }^{48}$ the 4 -Square Step Test

Table I Participant and Physiotherapist Interview Topics

\begin{tabular}{|l|}
\hline Introductory Question \\
\hline - Can you start by telling me a little bit about your stroke and how \\
has it affected your life since? \\
\hline Questions about the feasibility of the programme \\
(acceptability, implementation, practicality) \\
- How do you feel about the appropriateness of the activities/ \\
exercises/intensity of this programme? \\
- How do you feel about the suitability of this programme for (other) \\
patients after stroke? \\
- How do you feel about the practical side of things during this \\
programme? \\
- Have you ever had any safety concerns during the classes? \\
- Do you have any ideas about possible changes to the current \\
programme that would help other patients after stroke to complete it \\
in the future? \\
\hline Questions about personal changes after the programme \\
- Have you noticed any physical/mental/emotional/social changes \\
since participating in the programme? \\
- Have you noticed any changes in your independence/activities of \\
daily living since participating in the programme? \\
- Do you have any further suggestions? \\
\hline
\end{tabular}

$(\text { FSST) })^{49}$ and the 5-Times Sit-to-Stand Test (FTSTS). ${ }^{50,51}$ Specifically for this study, the stroke-specific Trunk Impairment Scale (TIS) ${ }^{52}$ was used to measure the motor impairment of the trunk through the evaluation of static and dynamic sitting balance as well as coordination of trunk movement. Two experienced physiotherapy lecturers (EB, LB) who were not involved in the programme sessions each performed half of all ten assessments in a random order.

\section{Data Analysis \\ Primary Outcomes}

Thematic analysis was used to analyse the interview transcripts and field notes. ${ }^{53}$ Data analysis employed both deductive and inductive approaches. ${ }^{43}$ Two researchers (JSGK, LDdJ) read through the anonymised interview transcripts and field notes independently several times to familiarise themselves with the data. Using Microsoft Word (Microsoft, 2016) the researchers independently organised preliminary codes identified using a deductive approach relevant to the participants' and the PTs' perspectives about the acceptability, implementation and practicality of the programme and the impact of participating in the programme on the participants' HRQoL-domains. ${ }^{43,54}$ Regarding the latter, an inductive approach was used to explore unanticipated codes and to identify deeper meanings. ${ }^{55}$ Codes were subsequently developed into more abstract and comprehensive units of understanding in the forms of subthemes, and initial candidate themes. ${ }^{55}$ Cross-examination between the researchers then commenced to achieve consensus around the subthemes and candidate themes. ${ }^{55}$ Together with a third researcher $(\mathrm{AMH})$, the candidate themes and overall data set were cross-examined until consensus was reached. This aimed to ensure an accurate representation of the data collected. Agreement on the final subthemes and themes was sought from all authors. Representative verbatim quotations were selected to exemplify the formation of subthemes and themes ${ }^{56}$ and labelled to indicate the participants' gender (M, F) and age or to indicate the quoted PTs (PT1, PT2). Final themes emerging from the interviews with the participants about their physical, mental, emotional and social well-being after participating in the programme were organised in a thematic map. This map illustrated the relationship between codes and themes, and was reviewed and refined such that they were all clearly reflected. ${ }^{55}$ Finally, a deductive approach was used to relate the overarching theme to the research question. This aimed to ensure that findings accurately reflected participants' perspectives 
regarding how participating impacted on the participants' health-related quality of life (HRQoL) in terms of their daily lived experience regarding physical, mental, emotional and social well-being.

\section{Secondary Outcomes}

Participant characteristics, including data on falls, slips and trips during the study period, were presented descriptively. Given the feasibility nature of the study, significance testing of the quantitative data collected (questionnaires, functional tests) was not performed ${ }^{57,58}$ and data were presented descriptively. These included median (interquartile ranges) SIS 3.0 domain scores, EQ-5D-5L (UK value set) index values, BBS, FES-I and TIS sum scores, as well as mean (standard deviations) times taken to perform the FTSTS, FSST and distances on the visual analogue scale (item 9 of the SIS).

\section{Assessment of the Feasibility to Progress to a Full Trial}

The a priori agreed progression criteria to judge the feasibility of a full trial were i) $\geq 70 \%$ of the participants completing at least 9 of the 12 group sessions and ii) $<10 \%$ of serious adverse events deemed due to the intervention.

\section{Results}

Participant characteristics are presented in Table 2. Four participants (80\%) attended all 12 programme sessions and one attended eight sessions. The two participants with the lowest BBS scores were the ones reporting one and five falls during the study period, respectively. None of these falls were injurious and most happened while the participants were dressing or moving in the dark. One participant fell during one of the exercises during one of the programme sessions but was also unharmed. No serious adverse events were reported.

An overview of the themes, subthemes and additional exemplary quotes emerging from the interviews with the five participants and the two PTs who delivered the programme about the acceptability, implementation and practicality of the programme are provided in Supplementary Data File 1 that can be found here: https://doi.org/10. 25917/5e 79caa8ead95

\section{Acceptability of the Programme}

The theme of acceptability captured the participants' and PTs' views about the novel programme in terms of appropriateness, satisfaction and safety. Overall, the participants strongly concurred that they enjoyed, and were satisfied with, the program for reasons of the fun, the camaraderie that developed and the friendly atmosphere during the sessions:

[...] everything I did I found it an adventure and it was fun. [...] A hell of a camaraderie develops amongst the team of people that you had out here, and I must say that that actually is like playing in a sports team. M (68)

The PTs enjoyed being able to help the participants achieve their goals and watch them improve their confidence and some of their functional capabilities:

Table 2 Characteristics of the Five Participating People After Stroke

\begin{tabular}{|c|c|c|c|c|c|}
\hline Characteristics & Participant I & Participant 2 & Participant 3 & Participant 4 & Participant 5 \\
\hline Age (years) & 67 & 57 & 73 & 70 & 68 \\
\hline Gender & Male & Female & Male & Male & Male \\
\hline Time since stroke (years + months) & $1+6$ & $2+4$ & $9+1$ & $9+11$ & $3+5$ \\
\hline Type of lesion & Haemorrhagic & Unknown & Haemorrhagic & Haemorrhagic & Ischemic \\
\hline Hemiplegic side & Left & Right & Unclear & Right & Left \\
\hline Mini Mental State Examination score & 30 & 29 & 29 & 30 & 30 \\
\hline Self-rated health status & Very good & Good & Good & Good & Very good \\
\hline Functional Ambulation Classification & $4^{\mathrm{a}}$ & $6^{\mathrm{b}}$ & 6 & 6 & 6 \\
\hline Number of different types of medications taken per day & 2 & $\mathrm{I}$ & 2 & 2 & 5 \\
\hline Previously discussed falls with your health provider? & Yes & Yes & Yes & Yes & Yes \\
\hline $\begin{array}{l}\text { Previously undertaken healthy ageing or falls prevention } \\
\text { activities? }\end{array}$ & Yes & Yes & Yes & Yes & No \\
\hline Number of falls, slips or trips in the past 12 months & 3 & 0 & I & 0 & 2 \\
\hline Injurious falls in the past 12 months? & Yes & No & Yes & No & Yes \\
\hline Number of falls, slips or trips during study period & 5 & 0 & 0 & c & I \\
\hline
\end{tabular}

Notes: ${ }^{\mathrm{a}} 4=$ independent ambulator. ${ }^{\mathrm{b}} 6=$ ambulator, but dependent for supervision. ${ }^{\mathrm{c}}$ Data missing. 
I really enjoyed watching their confidence improve and their functional capabilities improve as well over the 12 sessions, I really enjoyed it. Also [...] having that amount of time to spend with a client as well is really nice. So you get to know them and what they, what their goals are and being able to help them kind of navigate towards achieving some of those goals which I really enjoy doing. [...] So it touched on a lot of different areas to do with falls prevention. I think the participants got a lot out of it as well. I thought it was great. PT2

The participants felt that most of the different programme activities and exercises were appropriate for them and suitable for people after stroke:

To me that [education] set the scene. It was like theory in practice, yeah. No, I think that was brilliant, I loved that. M (73)

[The T'ai-Chi movements] were very easy, good movements and I must say I found that flow of movement was actually very nice. $\mathrm{M}(68)$

I think those [Exer]games are very good because what happens is that it gets me to do the stuff extensively and I think it actually creates more of a connection between my vision and my muscles which I think is very important. M (68)

[The falling techniques were] good fun [...] because I found it really interesting because that's the way I used to be. I used to throw myself around, like fall over and muck around and that was good because it was more me. [...] Very normal to fall over and fall over and make a fool of yourself and get up. M (70)

One PT specifically noticed how the participants enjoyed the T'ai-Chi exercises and also acknowledged the appropriateness of teaching the participants how to get on and off the floor:

I think it's very appropriate and I think that's one of the big things after having a stroke, is confidence. Getting on and off the floor and confidence surrounding falls and fear of falling and all that kind of thing. So I think it's very appropriate to target that towards stroke patients. PT2

Both participants and PTs reported some recommendations for improvement. These broadly related to the fine-tuning of the organisation, arranging a follow-up programme, but most notably to specific exercises:

When we combine the cognitive games with the exercise at the same time, then it's going to be difficult. I think I'd need to go back and have it simplified. [...] I'd say in the early stages [the cognitive Exergames are] definitely not [appropriate] Because [the exercise alone is] hard enough. M (73)

I think that they really enjoyed the T'ai-Chi though in the last few sessions, that might be interesting to incorporate that a little bit earlier on. PT2

With each session it becomes more clear that the falls technique training is not suitable for everybody. For example, $[\mathrm{M}(73)]$ has back complaints and he wears a corset which impedes him in his movement. [F (57)] is really afraid of falling and does not want to fall on the mat even from a very low height. In her case maybe a very slow progression could work. Field note, session 9.

None of the participants or PTs expressed any concerns for safety during the different activities and exercises.

\section{Implementation of the Programme}

The theme of implementation mainly captured positive opinions about how the new programme was delivered to the current group of people after stroke. It was mainly the PTs that offered their views for this theme. They felt positive about having been able to offer proper education, individual guidance and to tailor activities and exercises to individual participants despite it being offered in a group situation and despite some initial doubts about safety:

I do have to admit that in the beginning I was a bit nervous about even practicing any kind of falls techniques, thinking I was going to end up with an [injured participant]. PT1

Participants and PTs also reflected on resources that could be implemented if the programme was offered again in the future:

You have a very small number, only five people and you had two physios which you probably wouldn't get in a more practical situation, you wouldn't have that much staff available or prepared to release that staff, with an activity like that you might have one physio, one person with a screen and that would be about it, or an assistant or something. M (67)

The more external equipment you bring in makes it difficult to adapt for them to do at home. So yes, [...] part of what we were doing was therapy while we're here, but part of it was to do it at home. So you need to be able to be doing things in here that you can do at home rather than a lot of fancy different other things. So it's that fine line of what you're wanting to achieve. PT1 
I think the environment, perhaps, wasn't quite appropriate for practising [falling techniques] in that we didn't have nice crash mats that we could use. PT2

Both PTs and participants felt the venue and group space used during this study were suitable.

\section{Practicality of the Programme}

The theme of practicality captured some of the participant's and PTs' opinions about whether the programme could be carried out as delivered in the current form. Specific comments from two participants suggested that especially the Exergames were challenging to perform. Overall, the participants and PTs felt that the group size was appropriate and all participants were well able to follow the programme:

Nobody came in and said: "that was too easy". Nobody said: "I don't want to do as much". Sometimes you had to tell them to stop. I don't think anybody was held back at all. PT1

\section{Influence on the Participants' Daily Lived Experience}

The overarching theme identified was that participants felt empowered by participating in the programme. An overview of the themes, subthemes and additional exemplary quotes emerging from the interviews with the five participants about their physical, mental, emotional and social well-being after participating in the programme is provided in Supplementary Data File 2 (https://doi.org/10. 25917/5e79caa8ead95). The final conceptual framework relating to the HRQoL domains of physical, mental, emotional and social well-being (Figure 1) assisted to explain how participating in the programme changed the participants' daily lived experience.

\section{Physical Changes}

The participants' perceptions about physical changes were mixed. Two participants did not notice any physical improvements or improvements in their balance. Other participants perceived increases in strength, better balance, increased endurance or more normal gait after completing the program:

No [I haven't noticed any physical changes in my body since starting the program]. M (73)

Standing up from a low seated position, that improved immensely. That improved $100 \%$ if not more. M (68)

I can turn around a bit quicker and can maintain my balance in different environments in different parts of the house. [...] I don't think [participating in the programme] had any effect on the limitations of the stroke, but it has allowed me to get my balance better, I personally think that. $M(67)$

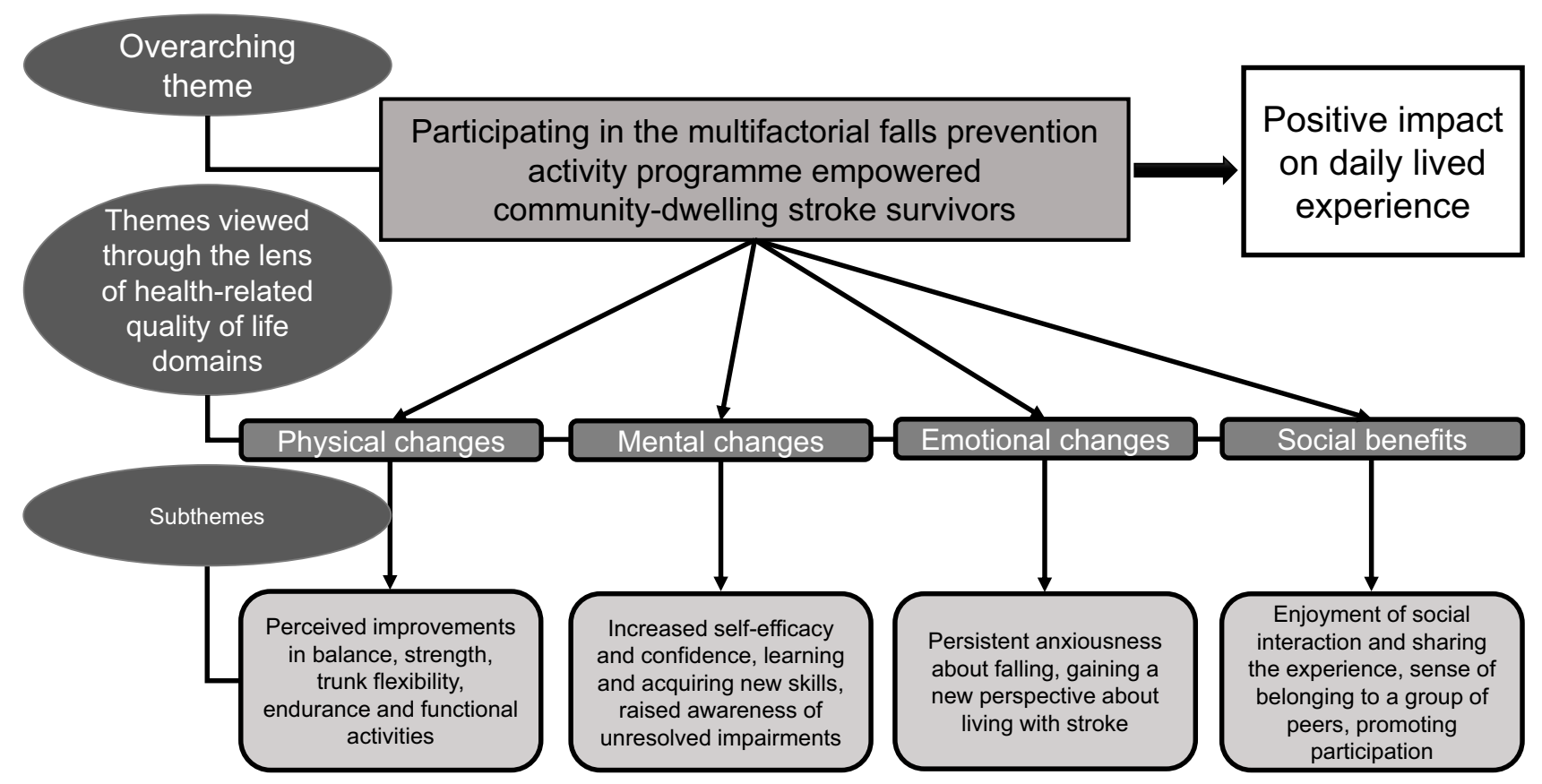

Figure I The final conceptual framework relating to the health-related quality of life (HRQoL) domains. 
Two participants perceived that participating in the programme increased their independence in some functional activities of daily life:

I always try to be as independent as possible to remove as much burden of stress on my wife, [...] The two things I do is I make my bed and clean my shoes myself. [...] It must have been since the program that I've started to do that. $M(68)$

Observations from the researchers supported these perceptions:

Participants were initially more willing to accept help from the research team when packing up at the end of classes but were observed to refuse similar help towards the end. Field note, session 10.

\section{Mental Changes}

Participants reported a range of mental changes, which suggested positive changes in self-efficacy and confidence:

I pushed my limitations a little bit more so than in the past. M (67)

[...] the more you do [the exercise], the more confident you get, and that confidence gives you an amazing feeling inside. M (68)

Additionally, some individual comments suggested that participants learned new skills or were made aware they had unresolved impairments in muscle functions. The latter empowered them to work towards their recovery beyond the confines of participation in the programme:

I'm trying to ask [the PTs] whether there was any of them could help me in the future because [...] I've recovered to almost look like I never had a stroke, but in fact, really, deep down I know I've got some issues with some of the muscles, muscle ability and that sort of thing. That's what had me. M (70)

I would continue doing all the stuff at home, you don't have to be in a class to do all of that but I continue doing that at home and I will be continuing. I haven't stopped the program, I haven't stopped doing what these activities suggest, I've still kept up to yesterday, so I'll continue with it, yeah. M (67)

\section{Emotional Changes}

Despite several positive mental changes, several participants remained anxious about falling:
I'm up in the sky here somewhere and if I lose my balance, I'm dead ... [...] I can't get that out of my brain. $M(73)$

On a more positive note, participating in the programme led some participants to gain a new perspective about living with stroke:

I also gained from the point of view that you're all the time comparing how am I going compared to some others. I figured out some people are worse than me, and I thought I was suffering pretty poorly from the stroke. M (67)

\section{Social Benefits}

Participants strongly concurred having enjoyed and valued the social element and interaction of the programme. One of the reported benefits was that participating in the programme instilled a sense of belonging to a group of peers, which offered an opportunity to share common experiences:

[...] chatting to the other guys and them telling me their stories was very, very nice to hear because then I relate, and I say oh you know something, that's what I had, and then the way a particular person got around that and how they would do it, that was very interesting. All those little tricks and tips is very, very important that everybody interacts and tells their story. That was very nice. M (68)

This participant also reflected on his interactions with healthcare professionals and carers during his own rehabilitation:

The thing that was missing, if I think about it now [...] was the social interaction. M (70).

Another participant felt the experience promoted his daily social interactions.

I think yes [participating in all these little gatherings that we have at the end of every session spurred me on to do more social activities outside of the program] because it shows that social activities are still very much a part of your life and [...] I try [...] going out to have coffee because I find that just interaction with that and something to look forward to doing. It stimulates your life and makes it worth living, so these little sessions show that there's still interaction between the people and everybody's got problems. It's not only your problems, so it's very good to have those sessions. M (68) 


\section{Secondary Outcomes}

Participants' quantitative data at baseline and after participating in the programme are presented in Table 3 . Overall, participants improved their balance, mobility, motor impairment of the trunk, strength and expressed less concerns over falling. The SIS domains of emotion and communication remained unchanged.

\section{Discussion}

The Fall Monty Activity Programme is a novel falls prevention activity programme designed to aid older people after stroke in functional recovery and reducing falls. In order to determine whether this programme could work, we set out to explore its feasibility in a small sample of community-dwelling older people in the chronic phase after stroke and with the two physiotherapists delivering the programme. The results of this study suggest that running the programme was feasible. In addition to the programme being feasible to run we noted that participants experienced positive changes in mental, emotional and social well-being and perceived improvements in their physical outcomes such as balance and strength. As such it may be appropriate to investigate the programme's efficacy and effectiveness in a randomised trial.
In the past decade, several multifactorial falls prevention programmes for older community-dwelling older people have been developed and evaluated. Only a limited number of studies have focussed on people after stroke. ${ }^{19,36}$ Unfortunately, most older people do not use falls prevention resources even when they are aware of them, ${ }^{59}$ for example because falls prevention is not perceived as being personally relevant to them. ${ }^{60}$ Therefore, this programme was intentionally framed as offering the participants a platform to learn how to prevent falls and giving them an opportunity to practice activities (rather than just offering exercises) that generally matter to people after stroke. ${ }^{61}$ In addition, a limited size group-based approach was chosen to offer the participants a safe environment to socialise and share thoughts and experiences with peers, and practice the different activities and exercises with one-to-one guidance if needed.

The quantitative results of this study suggest that participating in the programme may have positively influenced some of the participants' stroke-related impairments in body functions such as strength, balance and mobility. Participants made several comments supporting these results, especially relating to increased leg strength. These results contrasted the effects of another

Table 3 Participants' Quantitative (Physical) Outcomes at Baseline and After Participating in the 8-Week Activity Programme

\begin{tabular}{|c|c|c|}
\hline Outcome & Baseline & Outcome \\
\hline $\begin{array}{l}\text { BBS sum score, median }(\text { IQR) } \\
\text { EQ-5D-5L index value } \\
\text { FES-I sum score, median }^{\mathrm{b}}(\mathrm{IQR})^{\mathrm{c}} \\
\text { FTSTS, time (seconds) } \\
\text { FSST, time (seconds) }^{\mathrm{e}}\end{array}$ & $\begin{array}{l}49(45 \text { to } 52.5) \\
0.72(0.08) \\
10(7.5 \text { to } 12) \\
13(5.6) \\
28(19.6)\end{array}$ & $\begin{array}{l}53(45 \text { to } 55.5) \\
0.81(0.13) \\
8(7.5 \text { to } 12) \\
10.4(3.6) \\
23.9(18.4)\end{array}$ \\
\hline $\begin{array}{l}\text { SIS domain scores, median }(\mathrm{IQR})^{\mathrm{f}} \\
\text { Physical domain } \\
\text { Cognitive domain } \\
\text { Emotion domain } \\
\text { Communication domain } \\
\text { Participation domain } \\
\text { Visual Analogue Scale score }(\mathrm{cm})\end{array}$ & $\begin{array}{l}83(58.5 \text { to } 90.3) \\
89.3(78.6 \text { to } 96.4) \\
88.9(82 \text { to } 95.8) \\
100(98.2 \text { to } 100) \\
85.7(58.9 \text { to } 96.4) \\
81(8.9)\end{array}$ & $\begin{array}{l}91.8(56.2 \text { to } 97.4) \\
96.4(89.3 \text { to } 100) \\
88.9(76.4 \text { to } 97.2) \\
100(92.9 \text { to } 100) \\
92.9(41.1 \text { to } 96.4) \\
91.6(8.5)\end{array}$ \\
\hline TIS, sum score, median (IQR)g & 15 (13 to 19) & $18(16$ to 21$)$ \\
\hline
\end{tabular}

Notes: ${ }^{\mathrm{a} S}$ cores range from 0 to 56 where a higher score indicates better static and dynamic balance. ${ }^{\mathrm{b}}$ The index value ranges between 0 and I where a score closer to $\mathrm{I}$ indicates a better health state. ${ }^{\mathrm{C} S c o r e s}$ range from 7 to 28 where a higher score indicates more concern over falling. ${ }^{\mathrm{d}} \mathrm{A}$ shorter time indicates better functional mobility and strength. ${ }^{\mathrm{e}} \mathrm{A}$ shorter time indicates better balance. ${ }^{\mathrm{f}}$ The domain scores range from 0 to I00 and are calculated using the equation $((\text { mean item score }-1) / 4)^{*} 100$. Higher item scores indicate a lower level of difficulty experienced with the task. The physical domain comprises the original SIS domains of strength, hand function, ADL/IADL and mobility. The latter are aggregated into a composite because they are highly correlated. The majority of domain scores were not normally distributed. To facilitate comparisons the medians (interquartile ranges) were calculated for all domains. ${ }^{8}$ Scores range from 0 to 23 where a higher score indicates better static and dynamic sitting balance and coordination of trunk movement. All data are means (standard deviation) unless stated otherwise.

Abbreviations: BBS, Berg Balance Scale; EQ-5D-5L, European Quality of Life 5 Dimensions Health Questionnaire; FES, Falls Efficacy ScaleInternational; FTSTS, Five Times Sit-to-Stand Test; FFST, Four Square Step Test; SIS, Stroke Impact Scale 3.0; TIS, Trunk Impairment Scale. 
multifactorial falls prevention program delivered to community-dwelling stroke survivors, which reported minimal effect in improving physical function nor falls-related efficacy as compared to usual physiotherapy care. ${ }^{62}$ In light of our small sample of participants and the results of a recent systematic review, ${ }^{36}$ which found low to very low-quality evidence that exercises as part of a multi-component intervention reduce the rate of falls after stroke, our quantitative results should be interpreted with caution.

\section{Acceptability, Implementation and Practicality of the Programme}

The qualitative findings of this study suggest that the participants and the PTs delivering the programme deemed the overall programme activities and exercises feasible for people after stroke. Observations from the researchers and some comments from participants suggested that especially the cognitive Exergames and the safe landing and falls technique activities were quite challenging for some, but nevertheless appropriate, enjoyable and safe. The feasibility and safety aspects of the Exergames have been reviewed and confirmed previously ${ }^{63,64}$ and our results accord with those findings. Similar research evaluating safe landing and falls technique activities in older adults and patients after stroke is lacking and thus warranted. Discussions within the research team after the study led to the conclusion that individual tailoring of, and close supervision during, these specific activities are necessary if these activities are to remain part of the programme.

\section{Changes in the Participants' Daily Lived Experience}

The qualitative findings of this study show that participants perceived participating in the programme as an empowering experience. These findings suggest that a multifactorial falls prevention program, integrating activities that include a social element and promote social participation, can positively influence stroke survivors' daily lived experiences. This supports previous research which found exercise integrating peer-support elements enabled stroke survivors to positively reconstruct their lives after stroke. ${ }^{65,66}$ Alongside some perceived improvements in balance and strength, improvements on the FES-I suggested participants had less concern regarding the possibility of falling after the programme. It may be that the participant's increased knowledge and skill about how to land safely and get up from the floor contributed to an increase in confidence and self-efficacy. On the other hand, some participants indicated they were still anxious about falling. It would be worthwhile to investigate whether a longer duration programme could help to positively influence feelings of anxiety related to falling.

The individualised aspect of the program appeared to assist participants in gaining more awareness regarding their stroke recovery. This improved knowledge raised participants' self-efficacy, enabling them to incorporate strategies in their daily living to manage their deficits. We postulated that participants felt a renewed sense of confidence from having more control over their rehabilitation journey. This concurs with research reporting that stroke survivors who can independently manage their conditions had higher self-efficacy and less negative feelings about living with stroke. ${ }^{4,67}$ Self-efficacy was also improved by participants becoming more vigilant about environmental hazards and how these increase the risk of falls in the context of their physical deficits. Conducting the falls education and Exergames within a group setting also seemed to facilitate learning within and between participants, concurring with research on the benefits of peer support. ${ }^{68}$ Overall, our results are in line with results from a recent review which concluded that peer support facilitated shared experiences, social comparison, vicarious learning, and mutual gain. ${ }^{68}$ Various comments from our participants suggested that the interactions between them provided valuable opportunities to share and validate their respective lived experiences as a stroke survivor. For example, some comments suggested that it helped them to view their own disability through the lens of others who were worse off, allowing them to reduce their selfperceived level of disability. ${ }^{65,66}$ Similarly, stroke survivors who attended peer-support programs were found to have improved confidence, personal growth and enhanced social connections. ${ }^{69}$ Sharing their experiences facilitated our participants' empathetic and affirmative social engagement which was suggested to be missing from their usual day-to-day interactions with healthcare professionals and carers. The perception that healthcare practitioners lack the shared experience to fully understand their predicament was reported previously by stroke survivors. ${ }^{70}$ Our findings also reinforced how the presence of peer support facilitated joint problem solving, and may have helped in promoting social acceptance through generalising experiences to positively impact social wellbeing $^{68,69}$ as well as alleviating the sense of isolation many people after stroke feel. ${ }^{67}$ Taken together, these 
findings suggest that the integration of social elements within the rehabilitative experience for participants may improve physical recovery alongside improving social well-being and participation in societal and domestic roles. ${ }^{71}$ These benefits could be highlighted to pique older people's interest to engage in falls prevention activities $^{72}$ and used to promote programme adherence. ${ }^{73}$

\section{Strengths and Limitations of This Study}

Our study provided invaluable insights into the safety and appropriateness of delivering this novel falls prevention activity program for stroke survivors, which contributes to our current understanding of the topic and serves as a basis for continued work. The use of separate sources of data (older people after stroke, PTs' delivering the programme and researchers) allowed triangulation of the results to enhance the trustworthiness of our data analysis. The researcher diaries helped to improve reflexivity and minimised personal bias in influencing the data analysis process. The a priori progression criteria to judge the feasibility of progressing this programme to a full trial were met. The research team discussed the practicality of the study design, its impact on, and acceptability to, both older people after stroke and professionals. Given the participants' improvements in physical and health-related quality of life domains and the PTs' positive experiences with running the programme, progression of this novel programme to a randomised trial is currently being considered. Future research should also evaluate the entry requirements for this programme and the cost-effectiveness of this approach. In addition to research with older people after stroke, future research could also be carried out using other subgroups of participants with different levels of disability or a higher risk of falling. It may also be worthwhile to involve family members or carers in the programme. As this was a feasibility study using only five participants, generalisability of the results is limited. Our participants had a good to very good self-rated health status, were independently ambulant and on average over 5 years post-stroke. This allowed for undertaking activities and exercises at a level that might not be feasible for all people after stroke. Finally, thematic analysis of the data may not have reached saturation or sampled all important issues.

\section{Conclusion}

The overall results of this feasibility study suggest that running a novel multifactorial falls prevention activity programme for older community-dwelling people after stroke is feasible in terms of acceptability, implementation and practicality of the programme. The qualitative data suggest that community-dwelling stroke survivors felt participating in this group-based, multifactorial falls prevention activity program empowered them to make meaningful changes and positively improved their daily lived experiences. Raising personal self-efficacy by integrating multiple interventions, including a social element in a falls prevention activity program, may be integral in facilitating independence in the daily lives of stroke survivors. The quantitative data suggest that the programme may result in some improved strength and balance outcomes. A future fully powered study could build on these results to investigate physical improvements, prevention of falls and improvements to domains of HRQoOL.

\section{Data Sharing Statement}

De-identified participant data (quantitative outcomes, interview transcripts) and details about the content of the 12 sessions of our novel multifactorial falls prevention activity programme are available from the corresponding author on reasonable request up to 5 years following initial publication. Supplementary Data Files 1 and 2 are publicly available in the Research Data Australia repository, https:// doi.org/10.25917/5e79caa8ead95.

\section{Acknowledgments}

The authors would like to thank Trish Starling and Sally Wilson for running all programme sessions, and all participants for their participation.

\section{Disclosure}

MIRA Rehab Limited (London, UK) provided their Exergames software platform free of charge. The sponsor had no involvement in study design, data collection and analysis, decision to publish, or preparation of the manuscript. The authors report no other conflicts of interest in this work.

\section{References}

1. Mackay J, Mensah GA. The Atlas of Heart Disease and Stroke. Geneva: World Health Organization; 2004. 
2. Johnson CO, Nguyen M, Roth GA, et al. Global, regional, and national burden of stroke, 1990-2016: a systematic analysis for the global burden of disease study 2016. Lancet Neurol. 2019;18 (5):439-458. doi:10.1016/S1474-4422(19)30034-1

3. Weerdesteyn V, De Niet M, Van Duijnhoven HJR, Geurts ACH. Falls in individuals with stroke. J Rehabil Res Dev. 2008;45(8):1195-1214. doi:10.1682/JRRD.2007.09.0145

4. Pohjasvaara T, Vataja R, Leppävuori A, Kaste M, Erkinjuntti T. Depression is an independent predictor of poor long-term functional outcome post-stroke. Eur J Neurol. 2001;8(4):315-319. doi:10.1046/ j.1468-1331.2001.00182.x

5. Godwin KM, Ostwald SK, Cron SG, Wasserman J. Long-term health related quality of life of survivors of stroke and their spousal carers. J Neuroscience Nurs. 2013;45(3):147-154. doi:10.1097/ JNN.0b013e31828a410b

6. Sackley C, Brittle N, Patel S, et al. The prevalence of joint contractures, pressure sores, painful shoulder, other pain, falls, and depression in the year after a severely disabling stroke. Stroke. 2008;39 (12):3329-3334. doi:10.1161/STROKEAHA.108.518563

7. Mackintosh SFH, Goldie P, Hill K. Falls incidence and factors associated with falling in older, community-dwelling, chronic stroke survivors ( $>1$ year after stroke) and matched controls. Aging Clin Exp Res. 2005;17(2):74-81. doi:10.1007/BF03324577

8. Rigby H, Gubitz G, Phillips S. A systematic review of caregiver burden following stroke. Int $J$ Stroke. 2009;4(4):285-292. doi:10.1111/j.1747-4949.2009.00289.x

9. Ramnemark A, Nilsson M, Borsse B, Gustafson Y. Stroke, a major and increasing risk factor for femoral neck fracture. Stroke. 2000;31:1572-1577. doi:10.1161/01.STR.31.7.1572

10. Schmid AA, Arnold SE, Jones VA, Ritter MJ, Sapp SA, Van Puymbroeck M. Fear of falling in people with chronic stroke. Am J Occup Ther. 2015;69(3):6903350020. doi:10.5014/ajot.2015.016253

11. Goh HT, Nadarajah M, Hamzah NB, Varadan P, Tan MP. Falls and fear of falling after stroke: a case-control study. PM R. 2016;8 (12):1173-1180. doi:10.1016/j.pmrj.2016.05.012

12. Skelton D, Beyer N. Exercise and injury prevention in older people. Scand J Med Sci Sports. 2003;13(1):77-85. doi:10.1034/j.16000838.2003.00300.x

13. Sen A, Bisquera A, Wang Y, et al. Factors, trends, and long-term outcomes for stroke patients returning to work: the South London stroke register. Int J Stroke. 2019;14:1-10.

14. Woodman P, Riazi A, Pereira C, Jones F. Social participation post stroke: a meta-ethnographic review of the experiences and views of community-dwelling stroke survivors. Disabil Rehabil. 2014;36 (24):2031-2043. doi:10.3109/09638288.2014.887796

15. Azadeh H, Fekri A, Amraie H, Roostaei M, Baharlouei H. The correlation between rates of falling, balance, quality of life and fear of falling in patients with chronic stroke. J Rehabil. 2018;19 (1):36-43. doi:10.21859/jrehab.19.1.36

16. Paul SS, Lord SR, Michaleff ZA, et al. Exercise to prevent falls in older adults: an updated systematic review and meta-analysis. Br J Sports Med. 2016;51(24):1750-1758. doi:10.1136/bjsports-2016-096547

17. Van Duijnhoven HJR, Heeren A, Peters MAM, et al. Effects of exercise therapy on balance capacity in chronic stroke: systematic review and meta-analysis. Stroke. 2016;47(10):2603-2610. doi:10. 1161/STROKEAHA.116.013839

18. Hopewell S, Adedire O, Copsey BJ, et al. Multifactorial and multiple component interventions for preventing falls in older people living in the community. Cochrane Database Syst Rev. 2018;7.

19. Batchelor F, Hill K, MacKintosh S, Said C. What works in falls prevention after stroke?: a systematic review and meta-analysis. Stroke. 2010;41(8):1715-1722. doi:10.1161/STROKEAHA.109.570390

20. Skelton DA, Dinan SM. Exercise for falls management: rationale for an exercise programme aimed at reducing postural instability. Physiother Theory Pract. 1999;15(2):105-120. doi:10.1080/09593 9899307801
21. Iliffe S, Kendrick D, Morris R, et al. Multicentre cluster randomised trial comparing a community group exercise programme and home-based exercise with usual care for people aged 65 years and over in primary care. Health Technol Assess (Rockv). 2014;18 (49):1-105. doi:10.3310/hta18490

22. Thomas S, Mackintosh S, Does HJ. The "Otago exercise programme" reduce mortality and falls in older adults?: a systematic review and meta-analysis. Age Ageing. 2010;39(6):681-687. doi:10.1093/ageing/ afq102

23. Corbetta D, Imeri F, Gatti R. Rehabilitation that incorporates virtual reality is more effective than standard rehabilitation for improving walking speed, balance and mobility after stroke: a systematic review. J Physiother. 2015;61(3):117-124. doi:10.1016/j.jphys.2015.05.017

24. World Health Organization. WHO global report on falls prevention in older age. 2007. Available from: http://www.webcitation.org/ 6rKU08OH9. Accessed June 19, 2017..

25. Australian Commission on Safety and Quality in Health Care, Australian Commission on Safety and Quality in Healthcare. Preventing Falls and Harm from Falls in Older People; 2009.

26. Hill A-M, McPhail SM, Waldron N, et al. Fall rates in hospital rehabilitation units after individualised patient and staff education programmes: a pragmatic, stepped-wedge, cluster-randomised controlled trial. Lancet. 2015;385(9987):2592-2599. doi:10.1016/S01406736(14)61945-0

27. Luck T, Motzek T, Luppa M, et al. Effectiveness of preventive home visits in reducing the risk of falls in old age: a randomized controlled trial. Clin Interv Aging. 2013;8:697-702. doi:10.2147/CIA.S43284

28. Moon Y, Sosnoff JJ. Safe landing strategies during a fall: systematic review and meta-analysis. Arch Phys Med Rehabil. 2017;98 (4):783-794. doi:10.1016/j.apmr.2016.08.460

29. Moon Y, Bishnoi A, Sosnoff JJ, Shin JC, Un R. Preliminary investigation of teaching older adults the tuck-and-roll strategy: can older adults learn to fall with reduced impact severity. $J$ Biomech. 2018;83:291-297. doi:10.1016/j.jbiomech.2018.12.002

30. Groen BE, Smulders E, De Kam D, Duysens J, Weerdesteyn V. Martial arts fall training to prevent hip fractures in the elderly. Osteoporos Int. 2010;21(2):215-221. doi:10.1007/s00198-0090934-x

31. Harrison M, Ryan T, Gardiner C, Jones A. Psychological and emotional needs, assessment, and support post-stroke: a multi-perspective qualitative study. Top Stroke Rehabil. 2017;24(2):119-125. doi:10.1080/10749357.2016.1196908

32. Tse T, Binte Yusoff SZ, Churilov L, et al. Increased work and social engagement is associated with increased stroke specific quality of life in stroke survivors at 3 months and 12 months post-stroke: a longitudinal study of an Australian stroke cohort. Top Stroke Rehabil. 2017;9357(April):1-10.

33. Saunders DH, Greig CA, Mead GE. Physical activity and exercise after stroke: review of multiple meaningful benefits. Stroke. 2014;45 (12):3742-3747. doi:10.1161/STROKEAHA.114.004311

34. Reed M, Harrington R, Duggan Á, Wood VA. Meeting stroke survivors perceived needs: a qualitative study of a community-based exercise and education scheme. Clin Rehabil. 2010;24(1):16-25. doi: $10.1177 / 0269215509347433$

35. Ashburn A, Hyndman D, Pickering R, Yardley L, Harris S. Predicting people with stroke at risk of falls. Age Ageing. 2008;37(3):270-276. doi:10.1093/ageing/afn066

36. Denissen S, Staring W, Kunkel D, et al. Interventions for preventing falls in people after stroke. Cochrane Database Syst Rev. 2019;10.

37. Eldridge SM, Lancaster GA, Campbell MJ, et al. Defining feasibility and pilot studies in preparation for randomised controlled trials: development of a conceptual framework. PLoS One. 2016;11 (3):1-22. doi:10.1371/journal.pone.0150205

38. Orsmond GI, Cohn ES. The distinctive features of a feasibility study: objectives and guiding questions. OTJR Occup Particip Heal. 2015;35(3):169-177. doi:10.1177/1539449215578649 
39. Bowen DJ, Kreuter M, Spring B, et al. How we design feasibility studies. Am J Prev Med. 2009;36(5):452-457. doi:10.1016/j. amepre.2009.02.002

40. Kollen B, Kwakkel G, Lindeman E. Time dependency of walking classification in stroke. Phys Ther. 2006;86(5):618-625. doi:10.1093/ $\mathrm{ptj} / 86.5 .618$

41. Mehrholz J, Wagner K, Rutte K, et al. Predictive validity and responsiveness of the functional ambulation category in hemiparetic patients after stroke. Arch Phys Med Rehabil. 2007;88(10):1314-1319. doi:10.1016/j.apmr.2007.06.764

42. Lord S, Close JC, Ord STRL, Lose JACTC, Lord S, Close JC. New horizons in fall prevention. Age Ageing. 2018;47(April):1-7. doi:10.1093/ageing/afy059

43. Creswell J, Creswell J. Research Design: Qualitative, Quantitative, and Mixed Methods Approaches. 5th ed. Thousand Oaks, California: Sage Publications, Inc.; 2018.

44. Kempen GIJM, Yardley L, Van Haastregt JCM, et al. The short FES-I: a shortened version of the falls efficacy scale-international to assess fear of falling. Age Ageing. 2008;37(1):45-50. doi:10.1093/ ageing/afm 157

45. Herdman M, Gudex C, Lloyd A, et al. Development and preliminary testing of the new five-level version of EQ-5D (EQ-5D-5L) Qual Life Res. 2011;20(10):1727-1736. doi:10.1007/s11136-0119903-x

46. Duncan PW, Bode RK, Lai SM, Perera S. Rasch analysis of a new stroke-specific outcome scale: the stroke impact scale. Arch Phys Med Rehabil. 2003;84(7):950-963. doi:10.1016/S0003-9993(03) 00035-2

47. Mulder M, Nijland R. Stroke impact scale. J Physiother. 2016;62 (2):117. doi:10.1016/j.jphys.2016.02.002

48. Blum L, Korner-Bitensky N. Usefulness of the berg balance scale in stroke rehabilitation: a systematic review. Phys Ther. 2008;88 (5):559-566. doi:10.2522/ptj.20070205

49. Dite W, Temple VA. A clinical test of stepping and change of direction to identify multiple falling older adults. Arch Phys Med Rehabil. 2002;83(11):1566-1571. doi:10.1053/apmr.2002.35469

50. Whitney SL, Wrisley DM, Marchetti GF, Gee MA, Redfern MS, Furman JM. Clinical measurement of sit-to-stand performance in people with balance disorders: validity of data for the five-times-sitto-stand test. Phys Ther. 2005;85(10):1034-1045. doi:10.1093/ptj/ 85.10.1034

51. Mong Y, Teo TW, Ng SS. 5-repetition sit-to-stand test in subjects with chronic stroke: reliability and validity. Arch Phys Med Rehabil. 2010;91(3):407-413. doi:10.1016/j.apmr.2009.10.030

52. Verheyden G, Nieuwboer A, Mertin J, et al. The trunk impairment scale: a new tool to measure motor impairment of the trunk after stroke. Clin Rehabil. 2004;18(3):326-334.

53. Thomas E, Magilvy JK. Qualitative rigor or research validity in qualitative research. $J$ Spec Pediatr Nurs. 2011;16(2):151-155. doi:10.1111/j.1744-6155.2011.00283.x

54. Clarke P. Understanding the experience of stroke: a mixed-method research agenda. Gerontologist. 2009;49(3):293-302. doi:10.1093/ geront/gnp047

55. Braun V, Clarke V. Successful Qualitative Research: A Practical Guide for Beginners. 1st ed. London: SAGE Publications; 2013.

56. Polit D, Beck C. Essentials of Nursing Research. Appraising Evidence for Nursing Practice. 8th ed. Philadelphia: Wolters Kluwer, Lippincott Williams \& Wilkins; 2014.

57. Arain M, Campbell MJ, Cooper CL, Lancaster GA. What is a pilot or feasibility study? A Rev Curr Pract Editor Policy BMC Med Res Methodol. 2010;10:1-7.
58. Whitehead AL, Sully BGO, Campbell MJ. Pilot and feasibility studies: is there a difference from each other and from a randomised controlled trial? Contemp Clin Trials. 2014;38(1):130-133. doi:10.1016/j.cct.2014.04.001

59. McMahon SK, Park YS, Lewis MPHB, et al. Older adults 'utilization of community resources targeting fall prevention and physical activity. Gerontologist. 2018;1-11.

60. Dollard J, Barton C, Newbury J, Turnbull D. Older communitydwelling people's comparative optimism about falling: A populationbased telephone survey. Australas J Ageing. 2013;32(1):34-40. doi:10.1111/j.1741-6612.2012.00597.x

61. Segar ML, Guérin E, Phillips E, Fortier M. From a vital sign to vitality: selling exercise so patients want to buy it. Curr Sports Med Rep. 2016;15(4):276-281. doi:10.1249/JSR.0000000000000284

62. Batchelor FA, Hill KD, MacKintosh SF, Said CM, Whitehead CH. Effects of a multifactorial falls prevention program for people with stroke returning home after rehabilitation: a randomized controlled trial. Arch Phys Med Rehabil. 2012;93(9):1648-1655. doi:10.1016/j. apmr.2012.03.031

63. Skjæret N, Nawaz A, Morat T, Schoene D, Lægdheim J, Vereijken B. Exercise and rehabilitation delivered through exergames in older adults: an integrative review of technologies, safety and efficacy. Int J Med Inform. 2016;85:1-16. doi:10.1016/j.ijmedinf.2015.10.008

64. Adcock M, Thalmann M, Schättin A, Gennaro F, de Bruin ED. A pilot study of an in-home multicomponent exergame training for older adults: feasibility, usability and pre-post evaluation. Front Aging Neurosci. 2019;11(November):1-17. doi:10.3389/fnagi.2019. 00304

65. Kutlubaev MA, Hackett ML, Part II. Predictors of depression after stroke and impact of depression on stroke outcome: an updated systematic review of observational studies. Int J Stroke. 2014;9 (8):1026-1036. doi:10.1111/ijs.12356

66. Cho K, Lee G. Impaired dynamic balance is associated with falling in post-stroke patients. Tohoku J Exp Med. 2013;230(4):233-239. doi:10.1620/tjem.230.233

67. Hackett ML, Pickles K. Part I: frequency of depression after stroke: an updated systematic review and meta-analysis of observational studies. Int J Stroke. 2014;9(8):1017-1025. doi:10.1111/ ijs. 12357

68. Clark E, MacCrosain A, Ward NS, Jones F. The key features and role of peer support within group self-management interventions for stroke? A systematic review. Disabil Rehabil. 2018;42(3):1-10.

69. Kessler D, Egan M, Kubina LA. Peer support for stroke survivors: a case study. BMC Health Serv Res. 2014;14(1). doi:10.1186/14726963-14-256

70. Clark E, Bennett K, Ward N, Jones F. One size does not fit all-Stroke survivor's views on group self-management interventions. Disabil Rehabil. 2018;40(5):569-576. doi:10.1080/09638288.2016.1268653

71. Elloker T, Rhoda AJ. The relationship between social support and participation in stroke: a systematic review. African J Disabil. 2018;7:1-9. doi:10.4102/ajod.v7i0.357

72. Nyman SR, Victor CR. Older people's participation in and engagement with falls prevention interventions in community settings: an augment to the cochrane systematic review. Age Ageing. 2012;41 (1):16-23. doi:10.1093/ageing/afr103

73. de Jong LD, Coe D, Bailey C, Adams N, Skelton DA. Views and experiences of visually impaired older people and exercise instructors about the falls management exercise programme: a qualitative study. Disabil Rehabil. 2019;1-7. doi:10.1080/09638288.2019.1704894 


\section{Publish your work in this journal}

Clinical Interventions in Aging is an international, peer-reviewed journal focusing on evidence-based reports on the value or lack thereof of treatments intended to prevent or delay the onset of maladaptive correlates of aging in human beings. This journal is indexed on PubMed Central, MedLine, CAS, Scopus and the Elsevier
Bibliographic databases. The manuscript management system is completely online and includes a very quick and fair peer-review system, which is all easy to use. Visit http://www.dovepress.com/ testimonials.php to read real quotes from published authors. 